Article

\title{
Ionospheric Polarization Techniques for Robust NVIS Remote Sensing Platforms
}

\author{
Josep M. Maso $₫$, Jordi Male, Joaquim Porte $₫$, Joan L. Pijoan * ${ }^{\circledR}$ and David Badia \\ La Salle Campus, Ramon Llull University, 08022 Barcelona, Spain; josep.maso@salle.url.edu (J.M.M.); \\ jordi.male@salle.url.edu (J.M.); joaquim.porte@salle.url.edu (J.P.); david.badia@salle.url.edu (D.B.) \\ * Correspondence: joanlluis.pijoan@salle.url.edu
}

Received: 27 April 2020; Accepted: 26 May 2020; Published: 28 May 2020

check for updates

\section{Featured Application: Less power consumption, robust, and faster near vertical incidence skywave} remote sensing platforms.

\begin{abstract}
Every year more interest is focused on high frequencies (HF) communications for remote sensing platforms due to their capacity to establish links of more than $250 \mathrm{~km}$ without a line of sight and due to them being a low-cost alternative to satellite communications. In this article, we study the ionospheric ordinary and extraordinary waves to improve the applications of near vertical incidence skywave (NVIS) on a single input multiple output (SIMO) configuration. To obtain the results, we established a link of $95 \mathrm{~km}$ to test the diversity combining of ordinary and extraordinary waves by using selection combining (SC) and equal-gain combining (EGC) on a remote sensing platform. The testbench is based on digital modulation transmissions with power transmission between 3 and $100 \mathrm{~W}$. The results show us the main energy per bit to noise spectral density ratio $\left(\mathrm{E}_{\mathrm{b}} / \mathrm{N}_{0}\right)$ and the bit error rate (BER) differences between ordinary and extraordinary waves, SC, and EGC. To conclude, diversity techniques show us a decrease of the power transmission need, allowing for the use of compact antennas and increasing battery autonomy. Furthermore, we present three different improvement options for NVIS SIMO remote sensing platforms depending on the requirements of bitrate, power consumption, and efficiency of communication.
\end{abstract}

Keywords: HF; NVIS; SIMO; diversity combining; remote sensing platform

\section{Introduction}

The world's communications have gone from the communication between millions and millions of people to billions and billions of "things." The current networks are focused in urban areas with many devices (NB-IoT, Lora-Wan, Sigfox [1,2]) and people (3G, 4G, GSM). Although these networks are remarkably large, they experience difficulties in remote areas. The main obstacle for the development of these networks in isolated zones is the difficulty of reaching the installation terrain, often due to the complicated orography. In such situations, the only viable solution is satellite communication, which leads to a high-cost and complex infrastructure, making the transceiver unfeasible to be integrated with a remote sensing platform.

Although the satellite alternative seems notably solid, regions like the North and South Poles do not present coverage, thus making it difficult to get data from remote sensors. Some works [3-9] in high frequency (HF) communications validate the viability of ionospheric communications in remote areas [10]. This alternative proposes a new network for remote Internet of Things (RIoT) scenarios. Combining the ionosphere as a channel with HF (frequencies from 3 to $10 \mathrm{MHz}$ ) creates the near vertical incidence skywave (NVIS) channel. The proposed link makes a coverage of up to $250 \mathrm{~km}$ without the need of a line of sight when using an incidence angle from $70^{\circ}$ to $90^{\circ}$. All these aspects are 
essential to develop a network with a low economic cost and a straightforward installation, resulting in an interesting alternative to satellite communications.

Although there are some previous works related to the performance of this channel [3-9], this study shows how some physical characteristics of the ionosphere will improve the performance of the NVIS channel.

The ionosphere is defined by having different layers. When using it as a channel, the link is mainly established with the F2 layer and, during maximum solar incidence, with the E layer. As the ionization of the atmosphere is directly affected by the Sun, the star's variation makes the NVIS channel very unstable, leading to undesired fading, interferences, and link losses. This ionization also affects the refraction index, creating two characteristic waves, the ordinary and the extraordinary. Reflected waves present different polarizations, which can be used for applying diversity techniques to improve robustness and capacity.

The study of geoscience, when applied to engineering systems, allows us to develop a new telecommunications platform for remote areas and remote sensing applications. Analyzing the performance of the ionosphere as a telecommunication channel will contribute to upgrading the yield of this network for remote communications. This work proves that the understanding and use of the ordinary and extraordinary waves [11] from the F2 layer of the ionosphere [12] lead to an improvement in the NVIS channel's performance. Moreover, applying single input multiple output (SIMO) [13] techniques to circular polarized antennas [14] will allow the system to receive both characteristic waves simultaneously, thus further increasing the channel's yield.

Ben A. Witvliet in [15] and Yvon Erhel in [13] measured the correlation between the ordinary and extraordinary waves; the results stated an isolation of at least $13 \mathrm{~dB}$. Our work not only studies the behavior of the characteristic waves but also demonstrates that this channel de-correlation can lead to an improvement in the ionospheric link.

U. Umaisaroh in [16] studied the NVIS channel with a $2 \times 2$ MIMO link. An improvement of the communication's capacity is achieved, as it focused on the throughput improvement compared to a SISO environment. In contrast to this, our work focuses on the economic cost of the overall system and the transmitting power, which are aspects not studied in [13].

Reference [13] also showed how the capacity of the ionospheric channel is improved by using both waves, but all the tests were realized with a $500 \mathrm{~W}$ power amplifier. Although it proves that using both waves improves the performance of the channel, is not tested in a data transmission protocol for a low power consumption platform.

Finally, our work proposes a comparison with different diversity combining algorithms (selection combining and equal-gain combining), merging both waves to improve the performance and the robustness of the NVIS channel.

This article is organized as follows. In Section 2, we explain the bases of the NVIS ordinary and extraordinary waves and their use on polarization techniques. In Section 3, we describe the developed system used to perform the tests. In Section 4, we detail the frame design and the tests performed. In Section 5, we present the results obtained of the tests. Finally, Section 6 contains the conclusions achieved.

\section{NVIS Characteristics}

In this section we explain in more detail the principal characteristics of the NVIS propagation, especially those of the ordinary and extraordinary waves. Moreover, we explain the use of these ionospheric waves to perform polarization diversity and diversity combining.

\subsection{Ordinary and Extraordinary Waves}

NVIS communications are completely dependent on the ionosphere and its behavior to the Sun. As the ionization of the upper layers of the ionosphere is strongly dependent on the Sun's radiation, the ionosphere varies both daily and seasonally. 
Because the energy from the Sun varies both daily and seasonally, the ionosphere is never static. The Sun, for instance, has times of extreme activity that causes storms and severe disruption to the propagation. Moreover, the ionosphere's behavior is affected by even more parameters, such as the height and the mixture of gasses [17].

This chemistry that was just mentioned also directly affects the rate of ionization, just as the density of atoms and the intensity of radiation do. Some chemical reactions take place quickly, causing high ionization, while others are slower, resulting in less ionization [10].

All the ionization that was explained illustrates the ionosphere's interaction with radio waves. When a radio wave reaches the ionosphere, the electric field in the wave forces the electrons in the ionosphere into oscillation at the same frequency as the radio wave. Some of the radiofrequency energy is released due to this resonant oscillation, on which the oscillating electrons may either re-combine, thereby losing the radio energy, or re-radiate the original wave energy (refraction) [17].

The refraction phenomenon is fundamental in all ionospheric links, and it occurs because ionized molecules present free electrons that are able to vibrate in response to the radio signals reaching them. The behavior of each of these moving electrons resembles small dipole antennas. Radio waves are bent away from areas of high ionization because these areas have a lower refractive index. This bending effect reduces as the radio wave frequency increases, until a point where it suddenly stops, letting the waves pass on through (this value is known as the critical frequency) [17].

As a result to the electron's vibration, it is quite normal for a radio wave to have its polarization changed by the ionosphere. Furthermore, waves of different polarization can be refracted in different amounts. This leads to their return to Earth in two different rays: the ordinary and extraordinary rays, both with distinct properties [10]. Just as with reflections from other layers of the atmosphere or at other times, these rays can have different phases, amplitudes, frequencies, and times of arrival [17]. These different properties can be used in order to improve telecommunication links, as polarization diversity techniques are an option in ionospheric channels.

\subsection{Polarization Diversity and Diversity Combining}

Polarization diversity was firstly applied to HF, radar, and imaging systems. Its potential for improving the capacity of wireless communications systems was demonstrated, despite some disappointing premature predictions [18]. The improvement generated by this diversity method is typically granted by an additional de-correlated channel provided by a polarization state that is orthogonal to the existing one.

NVIS communications can clearly benefit from polarization diversity techniques due to one of these aspects: the two characteristic wave components with circular polarization of the opposite sense, both of which follow a different path through the ionosphere when a radio wave of a certain frequency reaches the ionosphere. The other key issue with evaluating parameters in this kind of scenario is cross-polarization discrimination (XPD), with low correlation co-efficients being achieved in most situations. Nevertheless, considerably more attention has been paid to spatial diversity due to the significant difference in the mean received signal level between the co-polarized and cross-polarized branches when one polarization is transmitted. Yet, spatial diversity is not the best solution after all, since at least $20 \lambda$ horizontal and $15 \lambda$ vertical separation distances are required for efficient outdoor diversity, resulting in an attraction gain for polarization diversity [19].

The implementation of a diversity system based on polarization needs some extra infrastructure compared to a rather simple scenario. As with any other diversity environments, multiple antennas are needed, specifically at the receiver. Both multiple input multiple output (MIMO) and SIMO are valid solutions, with the MIMO being the one that provides a better performance as it involves multiple antennas in the transmitter.

The effectiveness and success of both HF MIMO systems and diversity reception increases with the decrease of the correlation between the received signals. If the de-coupling between both characteristic waves is large enough and our system is correctly designed to combine the diversity 
correctly, these features will drastically improve HF MIMO and diversity reception. In [18], the behavior and the correlation of the two ionospheric characteristic waves were studied, where both signals achieved de-correlations that can reach values like $13 \mathrm{~dB}$ easily. Taking into account the propagation mechanism studied and the clear isolation (more than $12 \mathrm{~dB}$ ) between both characteristic waves, it is safe to state that a scenario that implements diversity reception can greatly benefit from the use of a pair of orthogonal circularly polarized antennas. Specifically, the use of right-hand circular polarization (RHCP) and left-hand circular polarization (LHCP) antennas for HF MIMO will create two very isolated channels that will maximize MIMO gain. In the study done in this article, though, the transmit antenna will be a linear polarized one while having two circular polarized antennas in the receiver.

All diversity techniques need a process for combining the different signals that arrive at the receiver. There are a lot of them, each one presenting different characteristics and gains. This article studies two different techniques: the selection combining, a method that selects the strongest signal received and discards the other, and the equal-gain combining, a technique that sums all the signals received coherently.

\section{System Description}

The main challenge of the performed tests is to analyze the influence of the ordinary and extraordinary ionospheric waves at the same time through an NVIS channel. Furthermore, the system requires us to analyze at the same time both ionospheric waves and apply polarization techniques to reach the best results on the demodulation of the frame. To achieve the goal, it is necessary to have a reconfigurable software defined radio platform.

One of the main disadvantages in the design of the transmission platform for an ionospheric link is having the ability to mitigate the effects of the channel. Due to new digital technologies, these processes are becoming more efficient and less costly. In addition, one of the main challenges is to develop a flexible platform that allows for the development of all the tests to be validated and for the implementation of all the tests and study hypotheses. All these requirements are solved with field-programmable gate array (FPGA) platforms.

To do this and to develop a low-cost platform, we have chosen the STEMlab 125-14 board hardware from the manufacturer Red Pitaya [20]. The main core of this hardware is a Zynq-7010-SOC [21]. These products contain a one-core (or two cores depending on which product is chosen) $A R M^{\circledR}$ Cortex ${ }^{\mathrm{TM}}$-A9 [22] based processing system (PS), plus $28 \mathrm{~nm}$ of Xilinx programmable logic (PL). In addition, this board has different peripherals (see in Figure 1) that allow us to make a robust platform for remote sensing:

- Zynq (FPGA/Cortex): The FPGA is essentially dedicated to data processing and the Cortex to time and data management. In the reception, this module allows us to receive and process the received signals in the analog digital converters (ADCs). This data is sent in real time to the Cortex via direct memory access (DMA). The Cortex is dedicated to saving the information on an external hard disk. In transmission, the Cortex contains the information that will be sent to the FPGA to be transmitted.

- Converters: The board has two channels, with two analog digital converters (ADCs), with a resolution of 14 bits and a sampling frequency of 125 MSPS. These, along with the two SMA connectors, will allow us to receive the signal by the antenna, send it to the FPGA, and treat the signal. The two ADCs allow us to receive both ordinary and extraordinary waves at the same time. This synchronization allows us to make a fair comparison with all the different polarization techniques. As opposed to ADC converters, digital analog converters (DACs) have a single converter to manage the two converter channels from the digital to the analog world; this fact should be considered when implementing the transmitter of the system. These will allow us to take out the signal already treated by the antenna. Therefore, this peripheral is only used by the FPGA [23]. 
- $\quad$ RaspBerry PI-3 [24]: This second post-processor is added to help the main core to control peripherals and save the received frames. The connection between both boards is realized via Ethernet.

- Amplifier: To carry out the various soundings with the FSK, QAM, and PSK modulations and with the transmissions of non-constant envelope modulations (QAM), using a class A amplifier is necessary. To carry out the study, an amplifier with a maximum power of $250 \mathrm{~W}$ was installed [25], although the maximum testbench power transmission is about $100 \mathrm{~W}$ and the minimum $3 \mathrm{~W}$.

- Filters: The platform contains two band-pass filters (BPF). The first transmitter is a band HF band-pass filter from 3 to $10 \mathrm{MHz}$. This frequency range is defined for the NVIS channel. The second one, is defined from 4 to $6 \mathrm{MHz}$ to filter possible interferences.

- GPS: The synchronization in time between the transmitter and the receiver is accomplished via GPS.

- $\quad$ Post-processing data: Finally, the post-processing of the data is performed by MATLAB software [26]. This is used to demodulate all the tests frames received and to obtain the results' graphics.

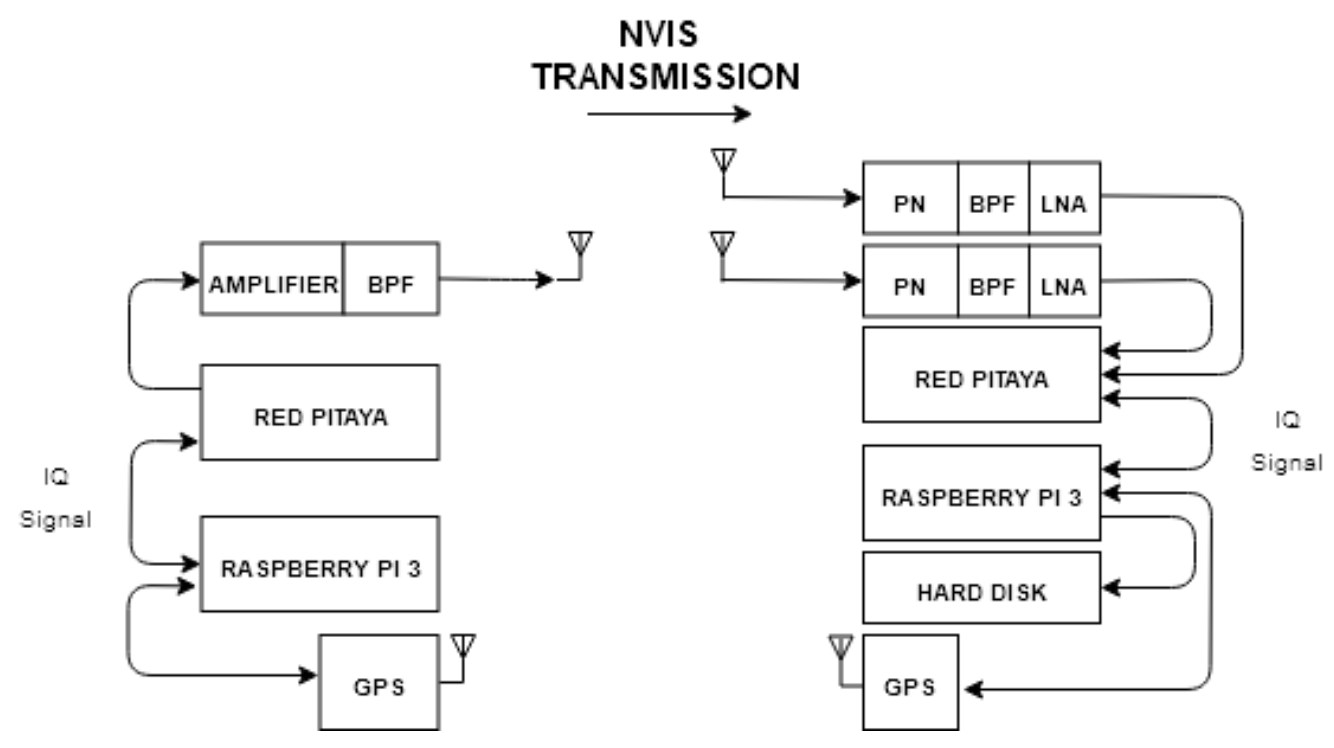

Figure 1. Schematic of the remote sensing platform.

The three antennas of the experiment are completely identical. All three are made of a flat copper conductor in the shape of inverted Vee and are tuned to the frequency of $5.40 \mathrm{MHz}$. The choice of the antenna design (inverted Vee) is based on its building facilities-they are easy to build as they only need a mast-and based on its gain, as it is similar to the horizontal dipole. Moreover, they are ideal for NVIS as they have a good radiation pattern in the upward direction.

The frequency was chosen and calculated from the revised ionograms of Observatori del Ebre [27]; this frequency is lower than the cutoff frequency as it allows us to correctly receive ordinary and extraordinary waves from the ionosphere. Concretely, and because of the study of the ionogram, it was possible to determine that the ideal frequency for the transmission to make the wave rebound with the minimum of possible losses is $10 \%$ less than the critical frequency F2.

The transmitter is less complex than the receiver as it only has one antenna. The reception part of the scenario is more sophisticated, as our experiment follows a single input multiple output design. The two reception antennas are set perpendicularly. This configuration together with a phasing network that delays one antenna from the other will allow the system to receive different polarizations at the same time, which is the main goal of the scenario itself.

A phasing box was designed and implemented to realize the delays mentioned with coaxial delay lines. The box gets a total of four wires, two from each antenna; we get two identical signals for each antenna with a radio frequency splitter. The paths of the two antennas are the same, as follows: one feed line is connected directly to a RF combiner, and the one cable is lengthened with a quarter 
wave phasing line to provide a $90^{\circ}$ phase shift. The output of the box gives us a phase difference between the dipoles antennas of either $+90^{\circ}$ and $-90^{\circ}$. For the designed system, the phasing network is for one single frequency. For a multiple frequency receptor, the phasing network should be done in digital. Figure 2 shows a diagram of the phasing network designed.

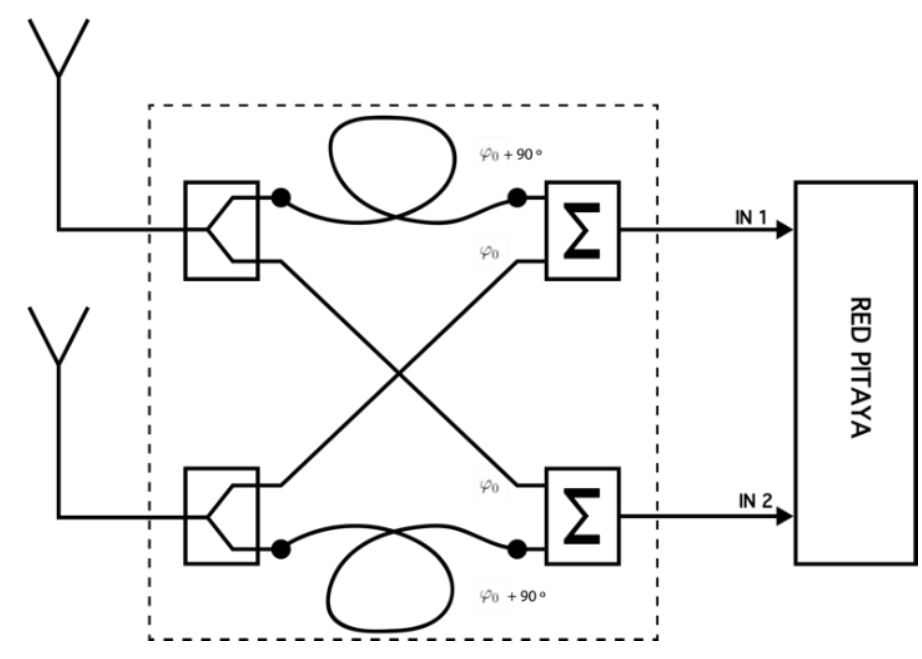

Figure 2. Diagram of the phasing network.

The goal of the phasing network is to unify the two antennas (initially linearly polarized and orthogonal to each other) so that two circularly polarized antennas are created. Specifically, the polarization vectors of the antennas are the following:

$$
\begin{aligned}
\operatorname{LHCP}_{\text {Antenna }} & =\hat{x}+j \cdot \hat{y}, \\
\operatorname{RHCP}_{\text {Antenna }} & =j \cdot \hat{x}+\hat{y},
\end{aligned}
$$

Equation (1) belongs to left-hand circular polarization, which corresponds to the ordinary wave in the Northern Hemisphere. Equation (2), the extraordinary wave, was studied, and it demonstrated that it has the lesser delay and RHCP in the Northern Hemisphere, while the ordinary wave has the greater delay and LHCP [18].

\section{Tests Performed}

In this section we show the scenario where we take the tests and the frame design of the signals transmitted.

\subsection{Test Scenario}

The performance tests were carried out in the Catalonia region (Spain), where we established a link between two NVIS nodes. The transmitter node was located at La Salle University-URL in Barcelona, and the receiver node was located at a remote location in Cambrils (Tarragona). In Figure 3, we can see the receiver node with two perpendicular inverted Vee antennas. The link between both locations is about $96 \mathrm{~km}$, ensuring an NVIS communication as we can see in Figure 4. The receiver was not set in Barcelona due to the high electromagnetic noise and interference at the HF band. For that reason, it was preferable to set the receiver in Cambrils. The tests performed were taken for 15 days from 7 December to 22 December 2019. The frames were transmitted every minute during these days. 


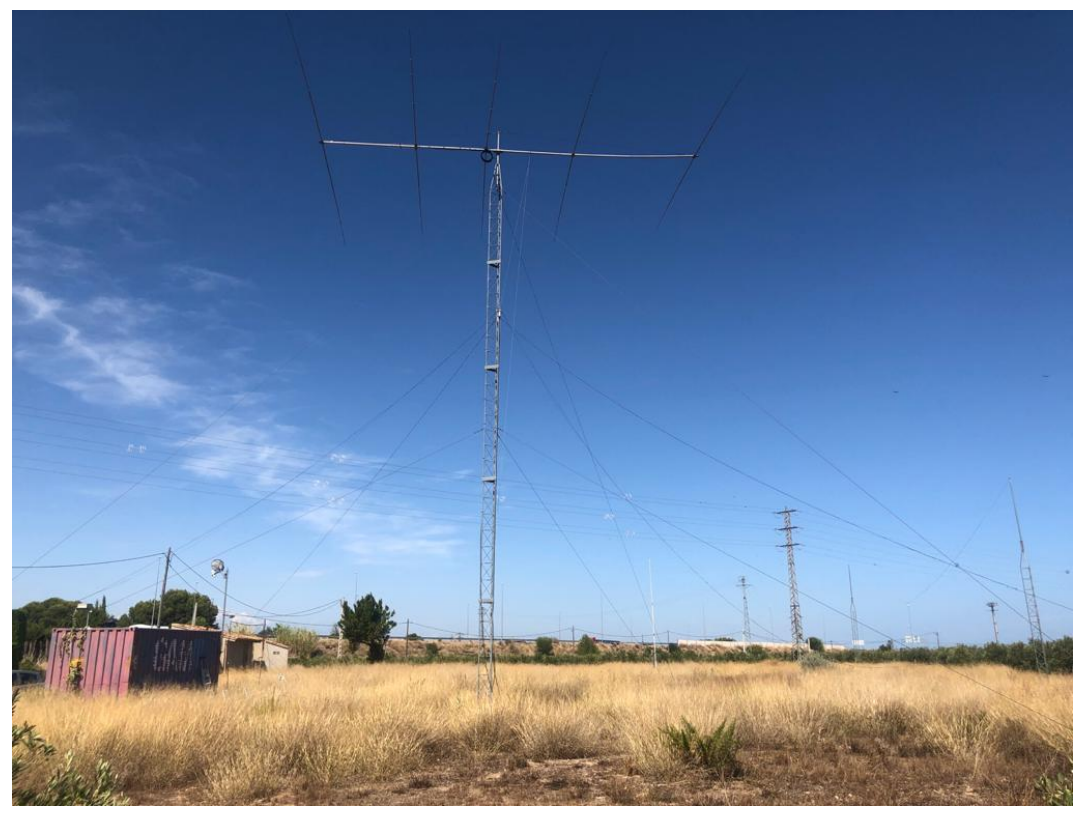

Figure 3. Receiver node in Cambrils (Spain).

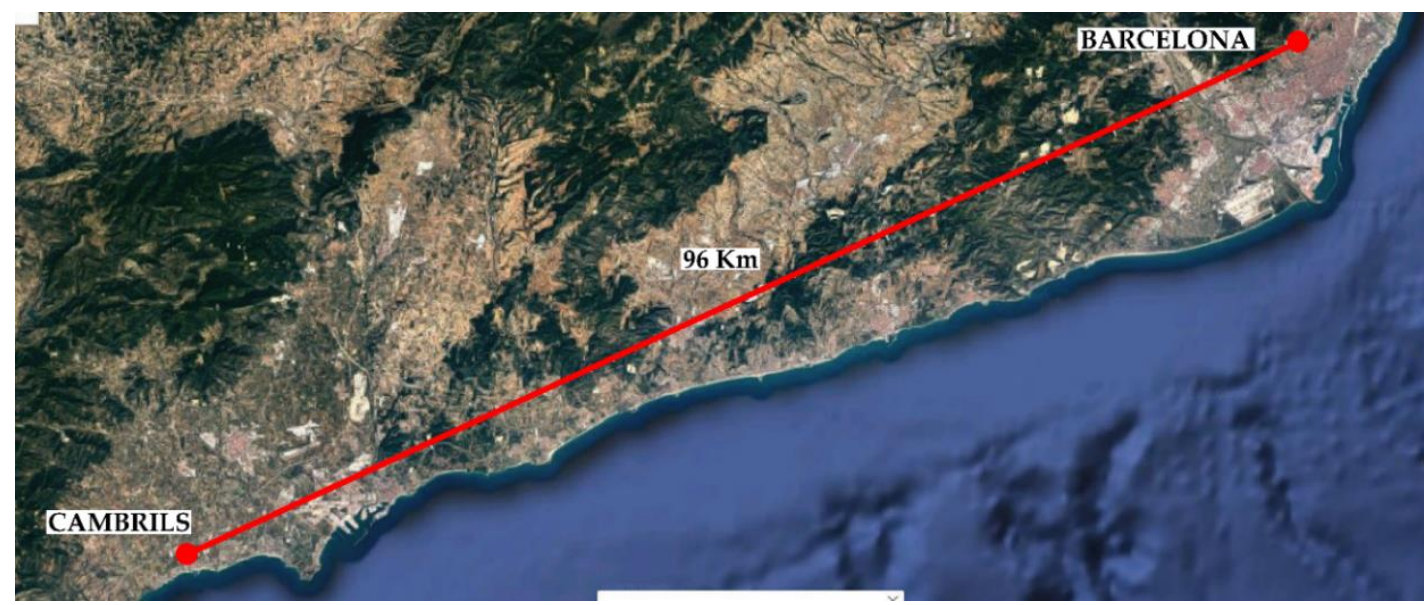

Figure 4. Near vertical incidence skywave (NVIS) test link.

\subsection{Frame Design}

To achieve the expected results in the tests, one of the most important parts of the study is the design of the frame to be transmitted. Every test consists of the transmission of the frame design in Figure 5 . The signal frame is composed of a total amount of 50 packets, and every packet is principally formed by three different modulations (PSK, FSK, and QAM). To synchronize the system with every modulation and to correct all the channel effects, every modulation is preceded by a $600 \mathrm{~Hz}$ tone and a PN sequence. In Figure 5, we can see that every data block contains 250 symbols with a resample of 42, resulting in a $2.3 \mathrm{kHz}$ bandwidth. Another important detail about the frame design is the time of each packet, which was carefully chosen to fulfill the coherence time requirements of the ionosphere [28]. Taking into account the most restrictive coherence time as $1.46 \mathrm{~s}$ [11], we set the time of the frame at $510.36 \mathrm{~ms}$. To compare the effects of the ordinary and extraordinary waves to the modulation transmitted, it is important to transmit every packet in the coherence time. The total duration of the 50 packets is about $25 \mathrm{~s}$.

Once the system identifies the start of the frame, the first block is the $600 \mathrm{~Hz}$ tone. This tone of a duration of $60 \mathrm{~ms}$ is a key issue for correcting the Doppler shift, which affects the rest of the block. The Red Pitaya platform clocks have a low stability, which creates a Doppler shift effect higher than the 
ionospheric. Our measurements of the platform show us that the maximum value of the Doppler shift received due to the low stability clocks is about $\pm 20 \mathrm{~Hz}$. The $600 \mathrm{~Hz}$ tone lets us identify the Doppler shift inserted by its variations between 580 and $620 \mathrm{~Hz}$. In the worst case, if we consider a received tone frequency of $580 \mathrm{~Hz}$, the measurement could be done with 34 cycles $(60 \mathrm{~ms})$. If we make use of a DC constant instead of a $600 \mathrm{~Hz}$ tone, the measurement will be done with the 16th part of a cycle (60 ms), which is not enough to take a real measurement.

The second block present on the frame is a 6th order PN sequence. The PN sequence is used for synchronizing with the modulations. The resampling of the PN sequence is about 8 and has a total duration of $5.12 \mathrm{~ms}$. The order and duration of the PN sequence were carefully designed to avoid the channel effects of a delay spread, Doppler spread, and Doppler shift.

Finally, the data blocks allow us to study the robustness of each modulation with different polarization techniques. The study performed was done with three different modulations (PSK, FSK, and QAM) with variating orders between 2 and 32 for power transmissions between 3 and $100 \mathrm{~W}$.
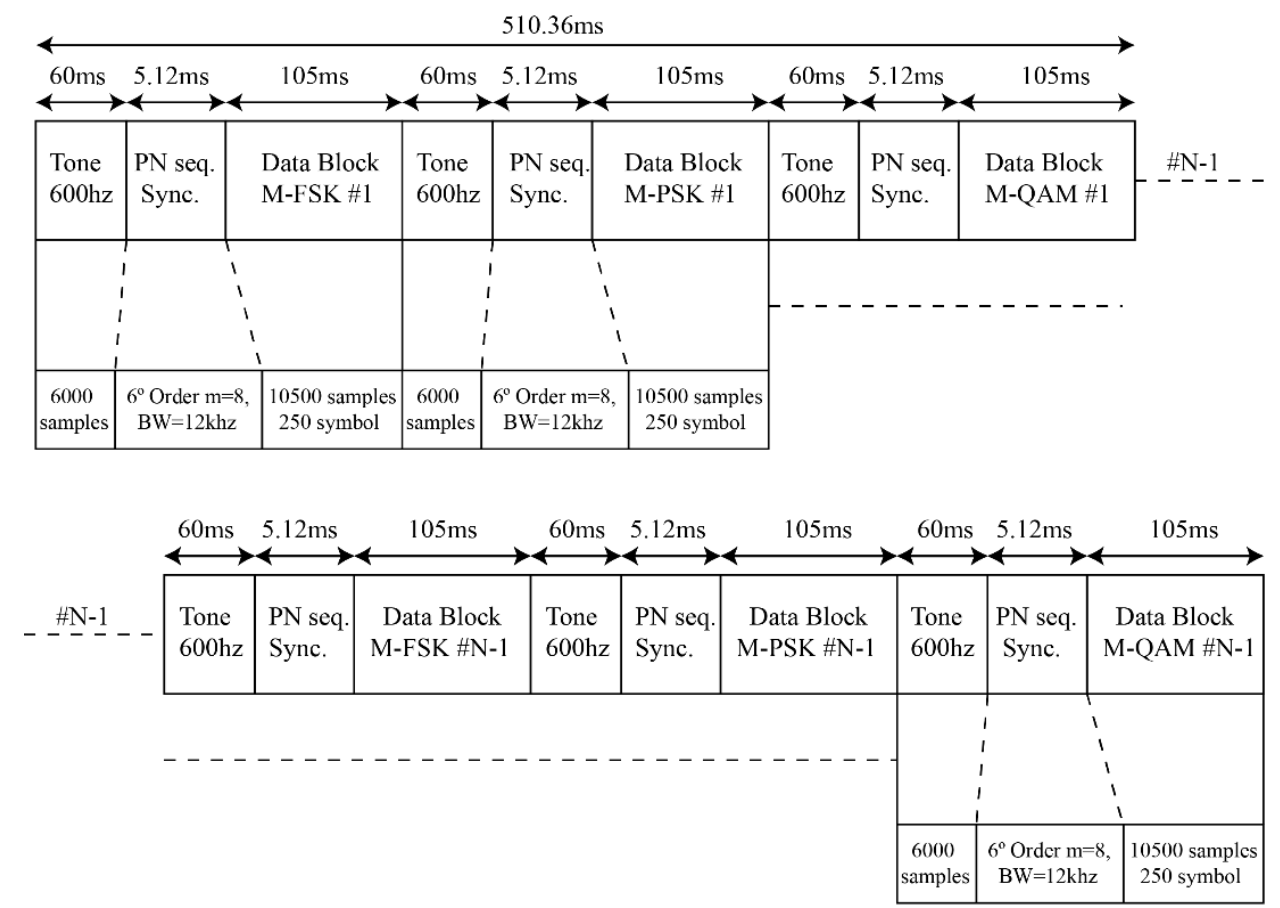

Figure 5. Frame design.

\subsection{Testbench}

The entire set of tests was classified by order modulations between 2 and 32 and power transmissions between 3 and $100 \mathrm{~W}$. For all of them, the bandwidth was set to $2.3 \mathrm{kHz}$, and we made use of three modulations (PSK, FSK, and QAM). After this, we obtain the results depending on the ionospheric layer reflected and depending on the polarization technique used by MATLAB post-processing. In one hour we perform 25 tests, as we can see in Table 1.

Table 1. Testbench transmitted.

\begin{tabular}{ccc}
\hline Order of Modulation & Power Transmission & Minute \\
\hline $2,4,8,16,32$ & $3 \mathrm{~W}$ & $05,06,07,08,09$ \\
$2,4,8,16,32$ & $6 \mathrm{~W}$ & $15,16,17,18,19$ \\
$2,4,8,16,32$ & $12 \mathrm{~W}$ & $25,26,27,28,29$ \\
$2,4,8,16,32$ & $25 \mathrm{~W}$ & $35,36,37,38,39$ \\
$2,4,8,16,32$ & $50 \mathrm{~W}$ & $45,46,47,48,49$ \\
$2,4,8,16,32$ & $100 \mathrm{~W}$ & $55,56,57,58,59$ \\
\hline
\end{tabular}




\section{Results}

In this section we analyze the most relevant results obtained from the tests performed. We will see the influence of the ordinary and extraordinary waves on the modulation's bit energy to noise power spectral density $\left(\mathrm{E}_{\mathrm{b}} / \mathrm{N}_{0}\right)$ and the effectiveness of the application of polarization techniques to obtain the best demodulation results. The tags "OR," "XOR," "SC," and "EGC" indicate the ordinary wave, the extraordinary wave, the selection combining, and the equal-gain combining, respectively.

\section{1. $E_{b} / N_{0} C D F$ According to Polarization Techniques}

First of all, we measured the relationship between the $E_{b} / N_{0}$ received from the ordinary and extraordinary waves and the $\mathrm{E}_{\mathrm{b}} / \mathrm{N}_{0}$ post-processed by the application of polarization techniques. The $E_{b} / N_{0}$ of the signal frame received is calculated as:

$$
\frac{E_{b}}{N_{0}}(d B)=\operatorname{SNR}(\mathrm{dB})+10 \cdot \log 10(B)-10 \cdot \log 10(R b),
$$

where the $\mathrm{Rb}$ is the bitrate of the signal (depending on the modulation order test), $\mathrm{B}$ is the noise bandwidth where we take the measurement of the bit error rate (BER) (which in our case is $2.3 \mathrm{kHz}$ ), and SNR is the signal noise ratio of the signal.

To analyze the relationship, we make use of the cumulative distribution function (CDF). In Figure 6, we can see the CDF where it represents the probability to receive a given $E_{b} / N_{0}$ value depending on whether it is received by the ordinary or extraordinary waves or by the application of a polarization technique. The $y$-axis shows us the probability $\mathrm{P}\left(\mathrm{E}_{\mathrm{b}} / \mathrm{N}_{0}<\mathrm{Xo}\right)$ of receiving an $\mathrm{E}_{\mathrm{b}} / \mathrm{N}_{0}$ less than or equal to value Xo of the $x$-axis. The graphic is obtained with the received signal of all power transmissions, which in this case is of a 4 QAM.

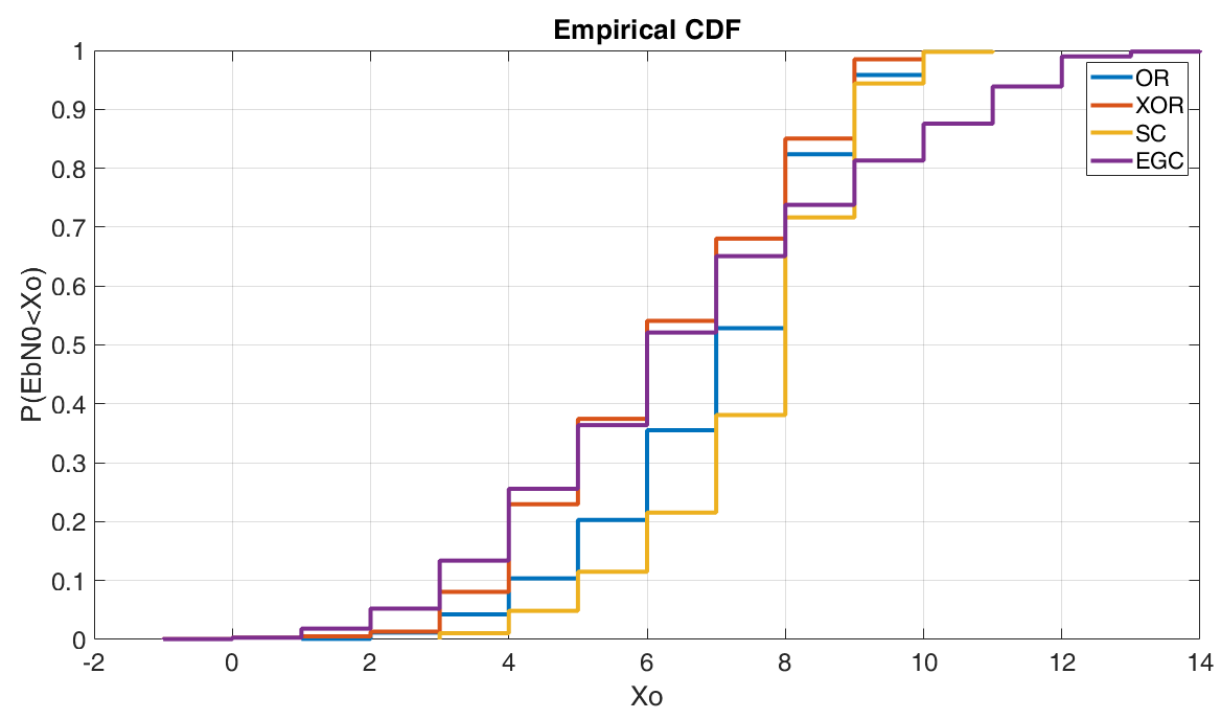

Figure 6. Bit energy to noise spectral density $\left(\mathrm{E}_{\mathrm{b}} / \mathrm{N}_{0}\right)$ cumulative distribution function $(\mathrm{CDF})$ of $4 \mathrm{QAM}$ at $50 \mathrm{~W}$.

First of all, we can see in Figure 6 that if we receive our signal by the ordinary and extraordinary waves, we have a slightly higher probability to receive a higher $E_{b} / N_{0}$ by the ordinary layer. In the case of using a polarization technique, the selection combining technique looks to be the best option to always receive the highest $\mathrm{E}_{\mathrm{b}} / \mathrm{N}_{0}$ of the ordinary and the extraordinary layers. In the worst case, with the equal-gain technique, depending on the electromagnetic noise, we can obtain the same $E_{b} / N_{0}$ as the lower $\mathrm{E}_{\mathrm{b}} / \mathrm{N}_{0}$ of the ordinary or extraordinary layer. In contraposition, in the best case, we can obtain $3 \mathrm{~dB}$ more than the selection combining technique in a probability of $1 \%$. Despite this low probability, 
we have a $20 \%$ probability to obtain a higher $\mathrm{E}_{\mathrm{b}} / \mathrm{N}_{0}$ than selection combining, as we can see in Figure 6 . Furthermore, we have a $35 \%$ probability to obtain the same results as selection combining.

\section{2. $E_{b} / N_{0}$ versus Time According to Polarization Techniques}

From another point of view, we can analyze the behavior of the ionospheric waves and the polarization techniques by the mean $\mathrm{E}_{\mathrm{b}} / \mathrm{N}_{0}$ received during different hours of the day. In Figure 7 , we can observe the results obtained by a $4 \mathrm{QAM}$ transmitted at $50 \mathrm{~W}$. As we can see, the $\mathrm{E}_{\mathrm{b}} / \mathrm{N}_{0}$ received by the ordinary and extraordinary waves differs by a maximum of $3 \mathrm{~dB}$. We can analyze that meanly, the selection combining $E_{b} / N_{0}$ is always higher than the $E_{b} / N_{0}$ received by the ordinary and extraordinary waves. Rarely, as we can see at 11 UTC, the mean of the selection combining is under one of the $E_{b} / N_{0}$ received mean values of an ionospheric layer. On the other hand, for $50 \%$ of the hours by equal-gain combining, we received a higher $\mathrm{E}_{\mathrm{b}} / \mathrm{N}_{0}$ than the ionospheric waves, and for $12 \%$ of the hours, a higher $E_{b} / N_{0}$ was received than by selection combining. For the other $50 \%$ of the hours, we received an $E_{b} / N_{0}$ between the received $E_{b} / N_{0}$ of the ordinary and extraordinary waves. On the other hand, we can analyze that the ordinary and extraordinary waves can have an $\mathrm{E}_{\mathrm{b}} / \mathrm{N}_{0}$ difference up to $3 \mathrm{~dB}$.

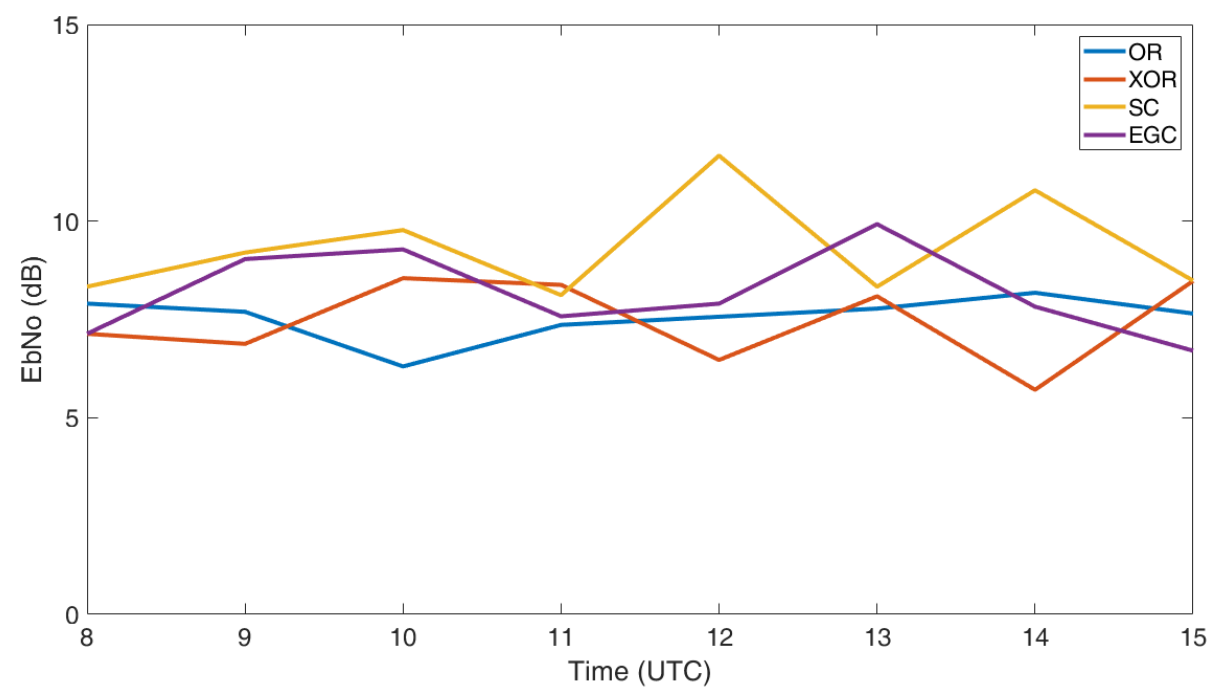

Figure 7. $\mathrm{E}_{\mathrm{b}} / \mathrm{N}_{0}$ vs Time for $4 \mathrm{QAM}$ at $50 \mathrm{~W}$.

\subsection{BER CDF According to Polarization Techniques}

Once we have analyzed the main behavior characteristics of the signal received in different ways, we can analyze the behavior of different orders and modulations received by ionospheric waves or polarization techniques. First of all, we analyze the modulations of order 4. In Figure 8, we can see that the ordinary and extraordinary waves are affected in the same way as the 4PSK and 4QAM with a 80\% probability to receive a BER lower than $10^{-4}$. In contrast, the 4 FSK has a $57 \%$ probability to receive a BER lower than $10^{-4}$. On the other hand, the selection combining looks to be the best option for the three modulations. To achieve a BER lower than $10^{-4}$, the 4 PSK has a $96 \%$ probability, the 4 FSK has a $88 \%$ probability, and the 4 QAM has a $85 \%$ probability. In the case of using equal-gain combining to achieve a BER lower than $10^{-4}$, the 4 PSK and 4QAM has a $82 \%$ probability and the 4 FSK has a $59 \%$ probability. For the BER CDF figures, we set a threshold at $10^{-4}$ because probabilistically we cannot ensure a BER lower than $10^{-4}$ due to the number of bits transmitted in all the test performed.

If we analyze the modulations of order 8 , we can note a decrease of the probabilities to achieve lower BERs because of the decrease of the $\mathrm{E}_{\mathrm{b}} / \mathrm{N}_{0}$ received. In Figure 9 , we can see that to achieve a BER lower than $10^{-4}$ by the ionospheric waves, for the 8PSK we have between a $42 \%$ and $55 \%$ probability, for the 8 FSK between a $8 \%$ and $26 \%$ probability, and the 8 QAM between a $1 \%$ and $24 \%$ probability. In this case, selection combining is still the best option for the 8PSK and 8FSK with a $70 \%$ and $40 \%$ probability, respectively, to achieve a BER lower than $10^{-4}$. For the $8 \mathrm{QAM}$, selection combining and 
equal-gain combining have the same results in this test, with a 39\% probability to achieve a BER lower than $10^{-4}$, which is the same for the equal-gain combining of $8 \mathrm{FSK}$. The $8 \mathrm{PSK}$ by equal-gain combining has a $50 \%$ probability to achieve a BER lower than $10^{-4}$.

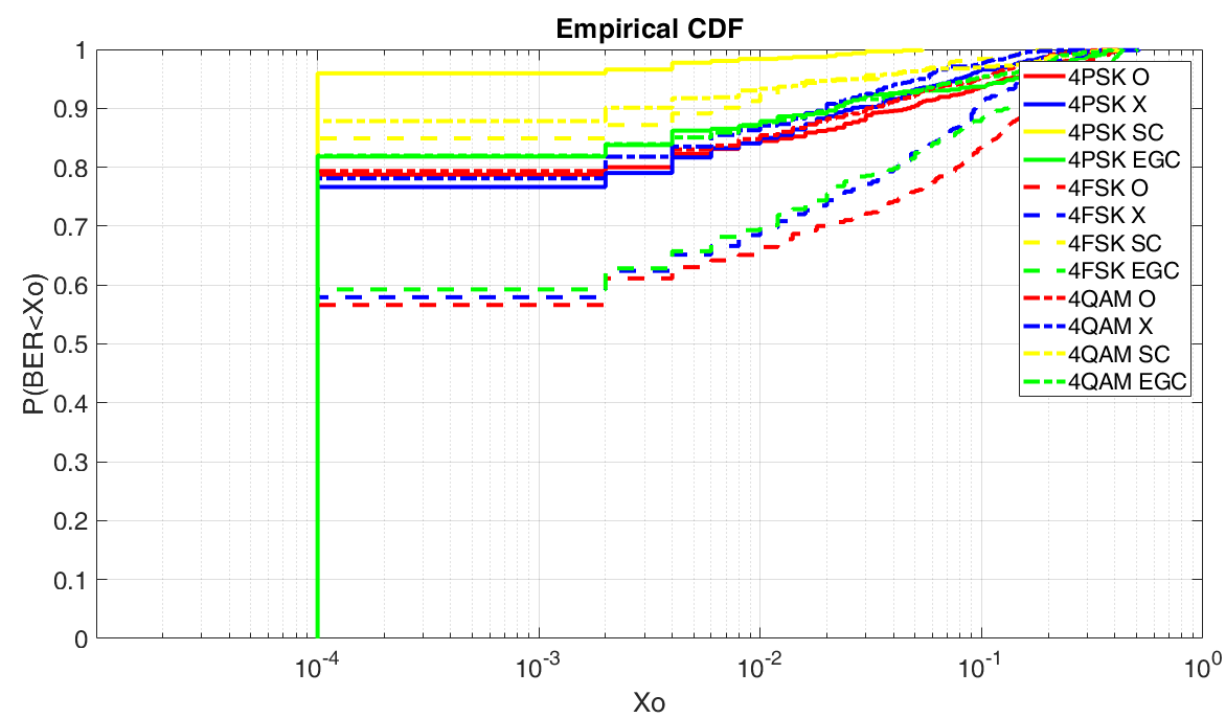

Figure 8. Bit error rate (BER) CDF order 4 modulations at $50 \mathrm{~W}$.

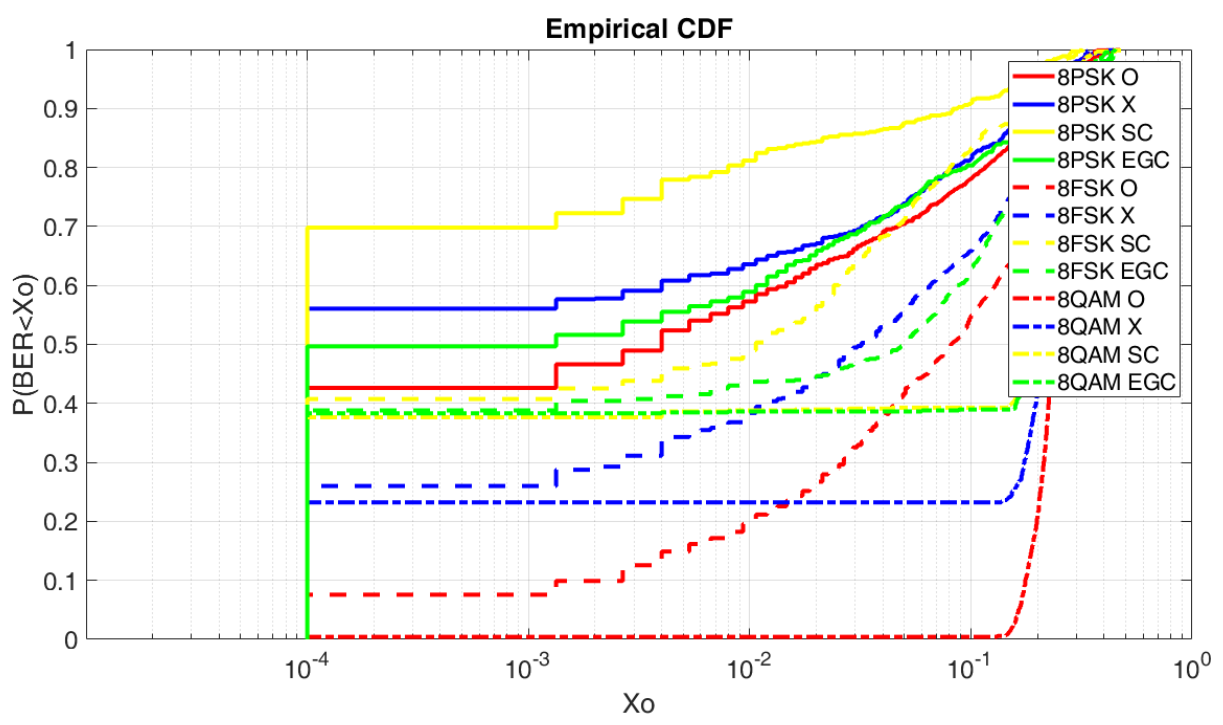

Figure 9. BER CDF order 8 modulations at $50 \mathrm{~W}$.

Despite having some good results for the 8PSK selection combining, when we increase the modulation order to 16 , the power transmission is not enough to achieve an $E_{b} / N_{0}$ that is enough to obtain a lower $\mathrm{E}_{\mathrm{b}} / \mathrm{N}_{0}$. As we can see in Figure 10, for 16QAM and 16FSK, to achieve a BER lower than $10^{-2}$ by the ionospheric waves, the probability is $1 \%$. The 16PSK is the best option in this case in order to obtain about a $7 \%$ probability. The selection combining for the 16PSK brings the best result of the graphic with a $25 \%$ probability to receive a BER lower than $10^{-4}$ and for equal-gain combining a $21 \%$ probability to achieve the same result. Furthermore, 16QAM and 16FSK obtain a great improvement by the use of selection combining and equal-gain combining with the same results for both polarization techniques, nearly a $20 \%$ probability to obtain a BER lower than $10^{-4}$. 


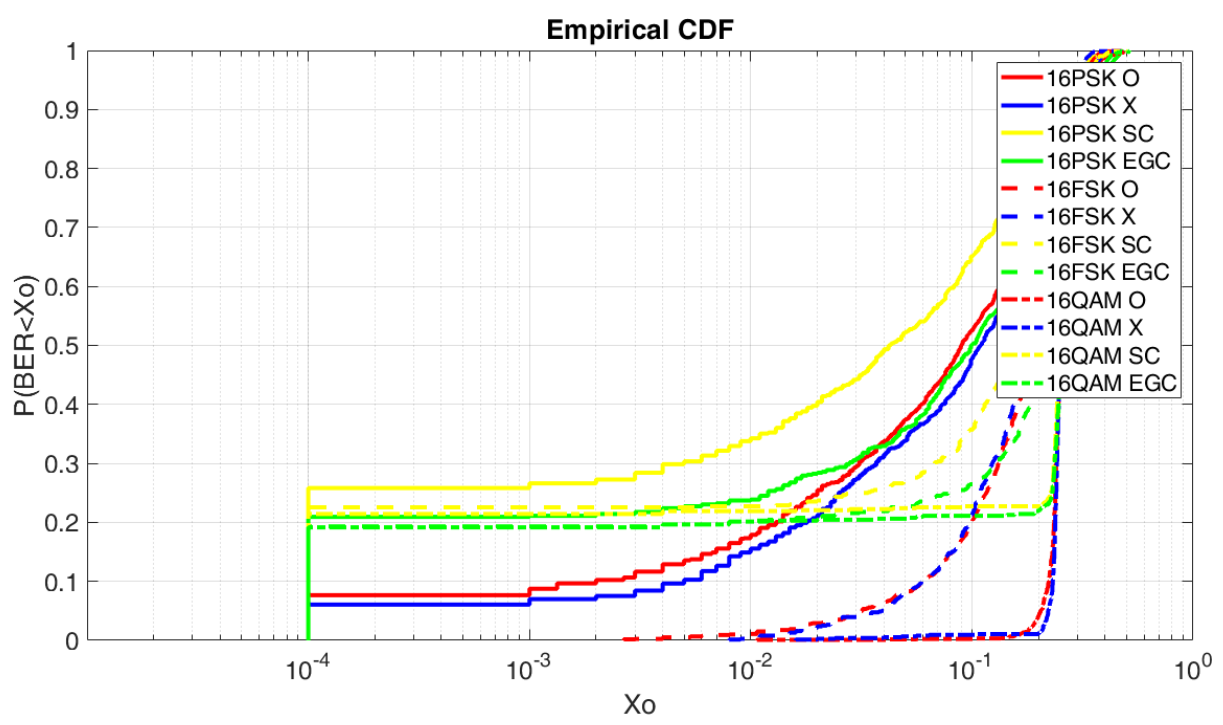

Figure 10. BER CDF order 16 modulations at $50 \mathrm{~W}$.

\subsection{BER CDF According to Power Transmission}

As we have seen, the power transmission of $50 \mathrm{~W}$ is not enough to obtain good results for higher modulation orders. The modulations of order 16 denotes a high decrease of the probabilities to achieve low BERs. Despite the results, we have seen that selection combining brings us the best results, especially for PSK modulation. However, a power transmission of $50 \mathrm{~W}$ is high, so taking into account these conclusions, we analyze the behavior of the PSK modulation orders 4 and 8 for a power transmission between $6 \mathrm{~W}$ and $50 \mathrm{~W}$. As we can see in Figure 11, the modulation 4PSK brings the best results in comparison to the 8PSK, regardless the power transmission. Even so, the bitrate of the 8PSK is higher. As the graphic shows, 4PSK with a power transmission of $50 \mathrm{~W}, 25 \mathrm{~W}, 12 \mathrm{~W}$, and $6 \mathrm{~W}$ have probabilities of $96 \%, 94 \%, 92 \%$, and $71 \%$, respectively, to achieve a BER lower than $10^{-4}$. The 8PSK with a power transmission of $50 \mathrm{~W}, 25 \mathrm{~W}, 12 \mathrm{~W}$, and $6 \mathrm{~W}$ have probabilities of $69 \%, 50 \%, 32 \%$, and $30 \%$, respectively, to achieve a BER lower than $10^{-4}$.

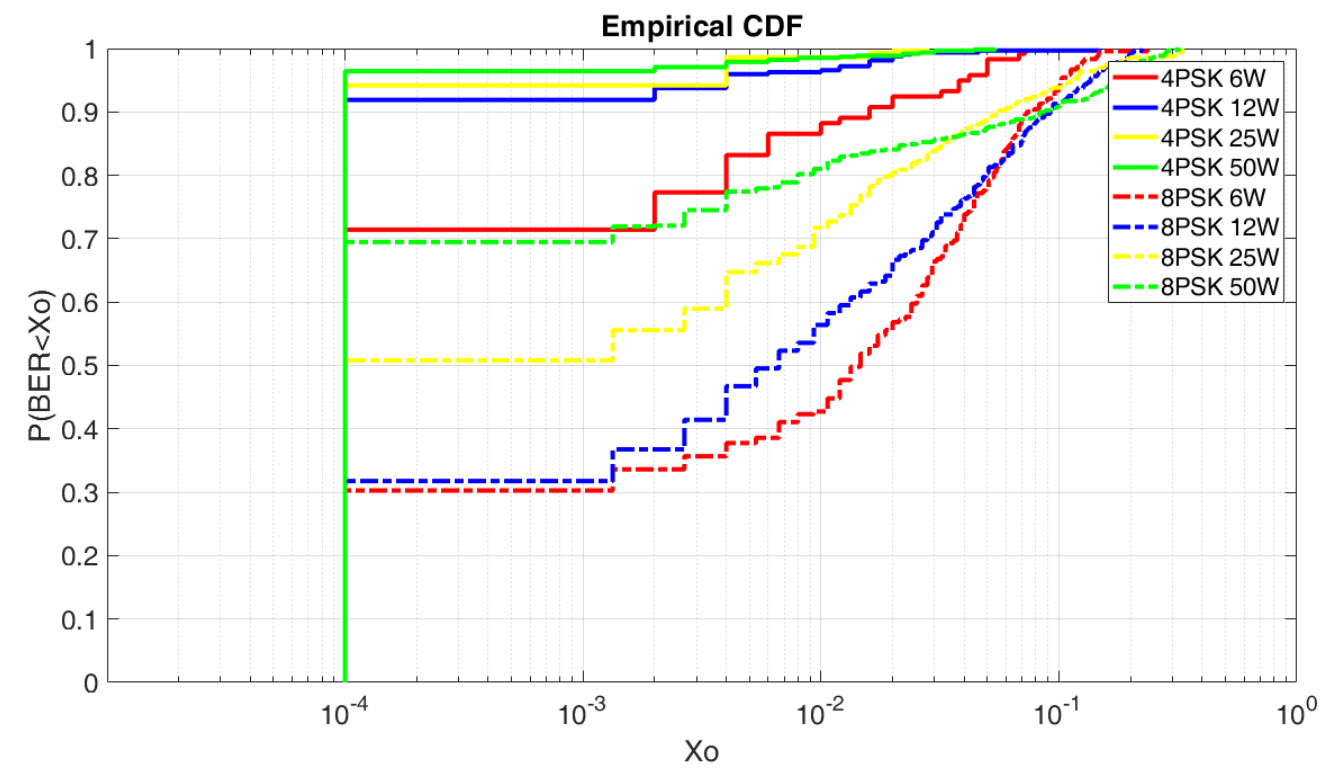

Figure 11. Selection combining (SC) BER CDF according to power transmission. 


\subsection{Number of Transmissions According to Selection Combining}

To form conclusions about which could be the best order modulation and power transmission to use with selection combining, we analyze, based on a Bernoulli distribution, the probability to receive a BER lower than $10^{-4}$ after several retransmissions. Analyzing the results in Figure 12. Number of transmissions according to selection combining, we can see that making use of a 4PSK with a power transmission of $12 \mathrm{~W}, 25 \mathrm{~W}$, and $50 \mathrm{~W}$, we have probabilities of $99 \%$ to achieve a BER lower than $10^{-4}$ if we retransmit the packet. In the case of using a 4PSK with a power transmission of $6 \mathrm{~W}$ or an 8PSK with a power transmission of $50 \mathrm{~W}$, we have nearly the same results. To achieve a $99 \%$ probability to obtain a BER lower than $10^{-4}$, it would be necessary to have 4 transmissions. With two transmissions, we can obtain probabilities of $91 \%$ to achieve a BER lower than $10^{-4}$, and with three transmissions, probabilities of $97 \%$ can be obtained to achieve a BER lower than $10^{-4}$.

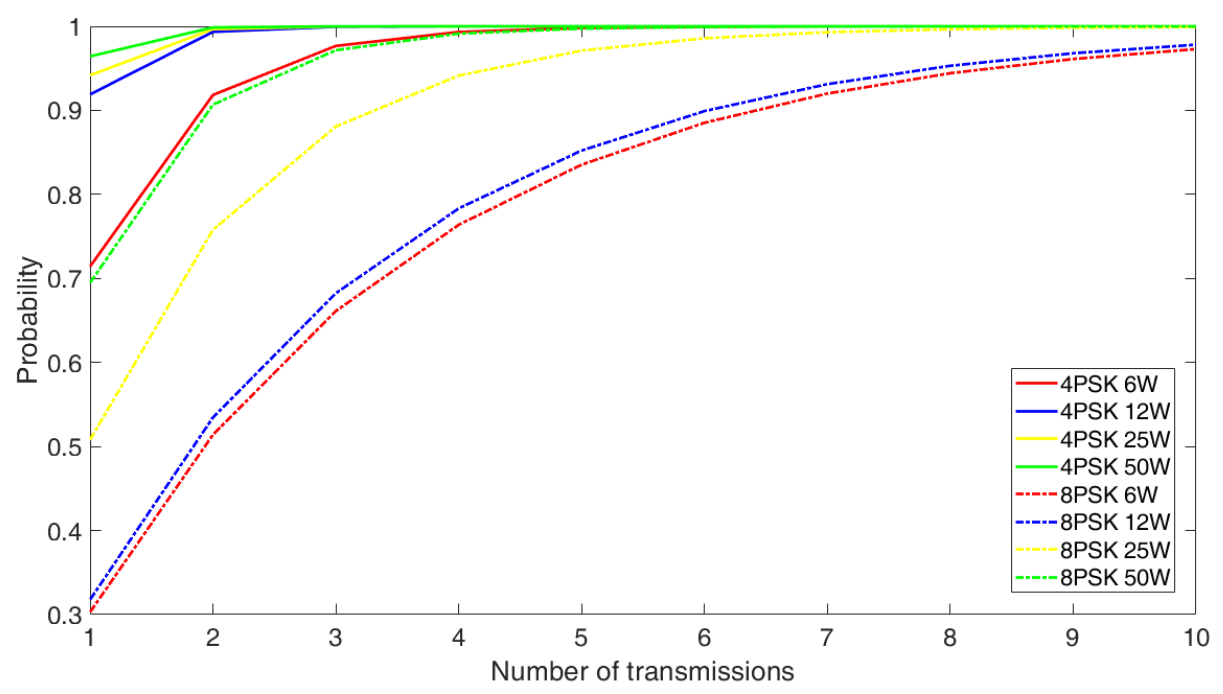

Figure 12. Number of transmissions according to selection combining.

\section{Conclusions}

After analyzing the results obtained, we observed that the ordinary and extraordinary waves are not received with the same $E_{b} / N_{0}$, which as we have seen can differ up to $3 d B$. These $E_{b} / N_{0}$ differences can provide us the possibility to apply polarization diversity techniques to take advantage of this fact.

First of all, we can conclude that selection combining helps us receive the highest $\mathrm{E}_{\mathrm{b}} / \mathrm{N}_{0}$ between ordinary and extraordinary waves. Consequently, by this polarization technique, we can receive meanly lower BER results. On the other hand, equal-gain combining is also a polarization technique that can obtain an $\mathrm{E}_{\mathrm{b}} / \mathrm{N}_{0} 3 \mathrm{~dB}$ higher than the highest $\mathrm{E}_{\mathrm{b}} / \mathrm{N}_{0}$ received by the ordinary and extraordinary waves. However, this possibility depends on the received electromagnetic noise, and it is limited to a $1 \%$ probability, but it is $20 \%$ probable to receive a higher $\mathrm{E}_{\mathrm{b}} / \mathrm{N}_{0}$ than the highest received by the ordinary and extraordinary waves.

Taking these results into account, if we make use of PSK, FSK, and QAM modulations orders between 2 and 32 with a power transmission of $50 \mathrm{~W}$, we analyzed that even with using polarization techniques, the BER obtained for modulations of order up to 16 is not low enough to ensure communication. Moreover, analyzing the results, we see that by PSK modulations with selection combining, we obtain the best BER results. For 4PSK and 8PSK modulations with selection combining and power transmissions variations between 6 and $50 \mathrm{~W}$, we can observe that the best BER results are similar for 4PSK with power transmissions between 12 and $50 \mathrm{~W}$. The second best BER results obtained are for 4PSK with $6 \mathrm{~W}$ and 8PSK with $50 \mathrm{~W}$.

In conclusion, we summarized in Table 2 three different options depending on the application and needs of the remote sensing system. 
Table 2. Application summary.

\begin{tabular}{ccccc}
\hline Power Consumption & Polarization Technique & Modulation & Bitrate & Probability to BER $\mathbf{~ 1 0}^{\mathbf{- 4}}$ \\
\hline $6 \mathrm{~W}$ & SC & $4 \mathrm{PSK}$ & $2.9 \mathrm{Kbps}$ & $71 \%$ \\
$12 \mathrm{~W}$ & SC & $4 \mathrm{PSK}$ & $2.9 \mathrm{Kbps}$ & $92 \%$ \\
$50 \mathrm{~W}$ & SC & $8 \mathrm{PSK}$ & $5.8 \mathrm{Kbps}$ & $70 \%$ \\
\hline
\end{tabular}

If our remote sensing system requires a low power usage, we can opt to use of a 4PSK with a power transmission of $6 \mathrm{~W}$. In this case, we have a $71 \%$ probability to receive a BER lower than $10^{-4}$ and a $99 \%$ probability if we make two retransmissions in the worst case. If our remote sensing system can make use of high power transmissions, the best option will be the usage of the 8PSK with $50 \mathrm{~W}$. This case will offer double the bitrate than 4PSK as well as a probability of 70\% to receive a BER lower than $10^{-4}$ and a probability of $99 \%$ if we make two retransmissions in the worst case. Lastly, if our remote sensing system requires low power consumption and needs to receive all packets with a minimum of retransmissions, we can choose a 4PSK with a power transmission of $12 \mathrm{~W}$. In this case, we have a $92 \%$ probability to receive a BER lower than $10^{-4}$ and a $99 \%$ probability if we make one retransmission. Moreover, the application of an error correction code (ECC) will highly increase the probabilities of receiving correctly the first packet transmitted. On the other hand, polarization techniques can compensate for the lower gains of compact antennas, making more feasible the use of them on HF communications. In addition, remote systems allow for using low power transmissions, which will decrease battery consumption and will make more feasible the use of renewable energies.

Author Contributions: Investigation, J.M.M., J.M. and J.P.; Methodology, J.L.P. and D.B.; Software, J.M.M., J.M. and J.P.; Supervision, J.L.P. and D.B.; Validation, J.L.P. and D.B.; Writing-original draft, J.M.M., J.M. and J.P.; Writing-review \& editing, J.M.M., J.M., J.P., J.L.P. and D.B. All authors have read and agreed to the published version of the manuscript.

Funding: This research was funded by the Spanish Ministry on Science, Innovation and University, the Investigation State Agency and the European Regional Development Fund (ERDF) under the grant number RTI2018-097066-B-I00 (MCIU/AEI/FEDER, UE) for the project "NVIS SENSOR NETWORK FOR THE SOUTH SHETLAND ISLANDS ARCHIPELAGO" (SHETLAND-NET).

Conflicts of Interest: The authors declare no conflict of interest.

\section{References}

1. Lauridsen, M.; Nguyen, H.; Vejlgaard, B.; Kovacs, I.Z.; Mogensen, P.; Sorensen, M. Coverage Comparison of GPRS, NB-IoT, LoRa, and SigFox in a $7800 \mathrm{~km}^{2}$ Area. In Proceedings of the 2017 IEEE 85th Vehicular Technology Conference (VTC Spring), Sydney, Australia, 4-7 June 2017; pp. 1-5.

2. Bechthum, E.; El Soussi, M.; Dijkhuis, J.; Mateman, P.; van Schaik, G.J.; Breeschoten, A.; Liu, Y.H.; Bachmann, C.; Philips, K. A CMOS polar single-supply class-G SCPA for LTE NB-IoT and cat-M1. In Proceedings of the ESSCIRC 2018-IEEE 44th European Solid State Circuits Conference (ESSCIRC), Dresden, Germany, 3-6 September 2018; pp. 30-33.

3. Porte, J.; Maso, J.M.; Pijoan, J.L.; Badia, D. Sensing system for remote areas in Antarctica. Radio Sci. 2020. [CrossRef]

4. Porte, J.; Maso, J.; Pijoan, J.L.; Miret, M.; Badia, D.; Jayasinghe, J. Education and e-health for developing countries using NVIS communications. In Proceedings of the 2018 IEEE Region 10 Humanitarian Technology Conference (R10-HTC), Malambe, Sri Lanka, 6-8 December 2018; pp. 1-5.

5. Porte, J.; Maso, J.M.; Pijoan, J.L.; Badia, D. Design, implementation, and test of an SDR for NVIS communications. Int. J. Circuit Theory Appl. 2019, 47, 1502-1512. [CrossRef]

6. Maso, J.; Porte, J.; Pijoan, J.L.; Badia, D. Internet of things communications for remote sensors in Antarctica using NVIS. HF Nordic. 2019. Available online: https://www.researchgate.net/profile/Joaquim_ Porte_Jimenez/publication/335774336_INTERNET_OF_THINGS_COMMUNICATIONS_FOR_REMOTE_ SENSORS_IN_ANTARCTICA_USING_NVIS/links/5d7a60354585157fde0fce47/INTERNET-OF-THINGSCOMMUNICATIONS-FOR-REMOTE-SENSORS-IN-ANTARCTICA-USING-NVIS.pdf (accessed on 26 May 2020). 
7. Porte, J.; Pijoan, J.L.; Masó, J.M.; Badia, D.; Zaballos, A.; Alsina-Pagès, R.M. Advanced HF communications for remote sensors in Antarctica. In Antartica-A Key to Global Change, 1st ed.; IntechOpen: London, UK, 2019; Available online: https://www.intechopen.com/books/antarctica-a-key-to-global-change/advanced-hfcommunications-for-remote-sensors-in-antarctica (accessed on 27 May 2020).

8. Orga, F.; Hervas, M.; Alsina-Pages, R.M. Flexible Low-Cost SDR Platform for HF Communications: Near vertical incidence skywave preliminary results. IEEE Antennas Propag. Mag. 2016, 58, 49-56. [CrossRef]

9. Wilson, J.M. A Low Power HF Communication System; Univesity of Manchester: Manchester, UK, 2012.

10. Davies, K. Ionospheric Radio; The Institution of Engineering and Technology (IET): Stevenage, UK, 1990.

11. Hervás, M.; Pijoan, J.L.; Alsina-Pagès, R.M.; Salvador, M.; Altadill, D. Channel Sounding and Polarization Diversity for the NVIS Channel. 2013. Available online: https://www.researchgate.net/profile/Marcos_Hervas/ publication/256375415_CHANNEL_SOUNDING_AND_POLARIZATION_DIVERSITY_FOR_THE_NVIS_ CHANNEL/links/0deec522664e6509e4000000/CHANNEL-SOUNDING-AND-POLARIZATION-DIVERSITYFOR-THE-NVIS-CHANNEL.pdf (accessed on 26 May 2020).

12. Witvliet, B.A.; Alsina-Pagès, R.M. Radio communication via Near Vertical Incidence Skywave propagation: An overview. Telecommun. Syst. 2017, 66, 295-309. [CrossRef]

13. Erhel, Y.; Lemur, D.; Oger, M.; Le Masson, J.; Marie, F. Evaluation of Ionospheric HF MIMO Channels: Two complementary circular polarizations reduce correlation. IEEE Antennas Propag. Mag. 2016, 58, $38-48$. [CrossRef]

14. Li, P.; Liang, C.H.; Tian, J. Polarization diversity experiments in HF communication. In Proceedings of the ISAPE 2003-2003 6th International Symposium on Antennas, Propagation and EM Theory, Proceedings, Beijing, China, 28 October-1 November 2003; pp. 556-559.

15. Witvliet, B.A.; Van Maanen, E.; Petersen, G.J.; Westenberg, A.J.; Bentum, M.J.; Slump, C.H.; Schiphorst, R. The importance of circular polarization for diversity reception and MIMO in NVIS propagation. In Proceedings of the 8th European Conference on Antennas and Propagation (EuCAP 2014), The Hague, The Netherlands, 6-11 April 2014; pp. 2797-2801.

16. Umaisaroh, U.; Hendrantoro, G.; Mauludiyanto, A.; Fukusako, T. Capacity of $2 \times 2$ MIMO HF NVIS Channels With Linearly Polarized Horizontal Antennas. IEEE Wirel. Commun. Lett. 2019, 8, 1120-1123. [CrossRef]

17. Greenman, M. An Introduction to HF propagation and the Ionosphere. QSL. Available online: https://www.qsl. net/zl1bpu/IONO/iono101.htm (accessed on 27 May 2020).

18. Witvliet, B.A. Near Vertical Incidence Skywave: Interaction of Antenna and Propagation Mechanism; Universiteit Twente: Enschede, The Netherlands, 2015.

19. Valenzuela-valdes, J.F.; Garcia-fernandez, M.A.; Martinez-gonzalez, A.M.; Sanchez-Hernandez, D. The Role of Polarization Diversity for MIMO Systems Under Rayleigh-Fading Environments. IEEE Antennas Wirel. Propag. Lett. 2006, 5, 534-536. [CrossRef]

20. Red Pitaya. Available online: https://www.redpitaya.com/ (accessed on 4 June 2019).

21. Xilinx and Inc. Zynq-7000 SoC First Generation Architecture. Available online: https://www.xilinx.com/ support/documentation/data_sheets/ds190-Zynq-7000-Overview.pdf (accessed on 27 May 2020).

22. Xilinx Zynq-7000 SoC ZC702 Evaluation Kit. Available online: https://www.xilinx.com/products/boardsand-kits/ek-z7-zc702-g.html (accessed on 20 January 2020).

23. Thomas, S.A.; Anusudha, K. Comparative analysis for various parametric attributes for an optimized DUC/DDC. In Proceedings of the 2017 International Conference on Inventive Computing and Informatics, ICICI 2017, Hotel Arcadia, Coimbatore, India, 23-24 November 2017; pp. 206-209.

24. Austin, R.; Bull, P.; Buffery, S. A raspberry Pi based scalable software defined network infrastructure for disaster relief communication. In Proceedings of the 2017 IEEE 5th International Conference on Future Internet of Things and Cloud, FiCloud 2017, Prague, Czech Republic, 21-23 August 2017; Volume 2017, pp. 265-271.

25. Elektronik, B. Bonn Elektronik Power amplifier 9 kHz-40 Ghz. Available online: https://allice.de/wp-content/ uploads/2017/03/Bonn-Katalog-Web.pdf (accessed on 27 May 2020).

26. R2015a-MATLAB \& Simulink-MathWorks España. Available online: https://es.mathworks.com/help/ matlab/release-notes-R2015a.html (accessed on 29 September 2019). 
27. Observatori de l'Ebre. Available online: http://www.obsebre.es/es/ (accessed on 21 February 2020).

28. Proakis, J.G. Digital Communications; McGraw-Hill: University of California, San Diego, CA, USA, 1995.

(C) 2020 by the authors. Licensee MDPI, Basel, Switzerland. This article is an open access article distributed under the terms and conditions of the Creative Commons Attribution (CC BY) license (http://creativecommons.org/licenses/by/4.0/). 